\title{
INFLUENCE OF POROELASTICITY ON THE 3D SEISMIC RESPONSE OF COMPLEX GEOLOGICAL MEDIA
}

\author{
Frank WuttKe ${ }^{1}$, Petia Dineva ${ }^{2}$, \\ IOANNA-KLEONIKI FONTARA ${ }^{3 *}$, \\ ${ }^{1}$ Institute of Applied Geoscience, Kiel University, Germany \\ ${ }^{2}$ Institute of Mechanics, Bulgarian Academy of Sciences, Sofia, Bulgaria \\ ${ }^{3}$ Institute of Civil Engineering, Technical University of Berlin, Germany
}

[Received 21 April 2017. Accepted 26 June 2017]

\begin{abstract}
Elastic wave propagation in 3D poroelastic geological media with localized heterogeneities, such as an elastic inclusion and a canyon is investigated to visualize the modification of local site responses under consideration of water saturated geomaterial. The extended computational environment herein developed is a direct Boundary Integral Equation Method (BIEM), based on the frequency-dependent fundamental solution of the governing equation in poro-visco elastodynamics. Bardet's model is introduced in the analysis as the computationally efficient viscoelastic isomorphism to Biot's equations of dynamic poroelasticity, thus replacing the two-phase material by a complex valued single-phase one. The potential of Bardet's analogue is illustrated for low frequency vibrations and all simulation results demonstrate the dependency of wave field developed along the free surface on the properties of the soil material.
\end{abstract}

KEY WORDS: 3D seismic wave propagation; saturated soil, surface relief, elastic inclusion, Boundary Integral Equation Method (BIEM).

\section{INTRODUCTION}

To date, it has proven impossible or technically expensive to consider important mechanical characteristics of geological materials as anisotropy, poroelasticity, inelasticity, inhomogeneity and heterogeneity in design codes, e. g. CEN Eurocode 8 [1]. This is due to several reasons, the primary one being the sheer complexity of the mechanical models, describing the seismic scenarios that may be developed at a given geographical location. Propagation of seismic waves through heterogeneous and inhomogeneous geological structures causes reflection, refraction, diffraction and scattering phenomena, that are difficult to quantify.

Different types of heterogeneities, such as buried cracks, cavities, inclusions, surface and subsurface topography, sedimentary basins complicate the overall picture

*Corresponding author e-mail: fontara@tu-berlin.de 
generating large amplification, as well as spatial variations in the seismic motions. In particular, the porous structure containing fluids modify the wave field behaviour drastically. All these have important repercussions in the analysis of large infrastructure such as dams, bridges, pipelines, tunnels, etc. The literature is quite rich in terms of results obtained for 2D wave scattering problems by different types of heterogeneities in homogeneous [2-6], discrete inhomogeneous [7] or continuously inhomogeneous continua [9-10]. The three-dimensional nature of the seismic motion doesn't simplify the modelling and it is also not taken into consideration in the design codes. Our attention in this work focuses on modelling of 3D seismic wave propagation in a heterogeneous poroelastic region, considering the influence of porosities, densities and topographical peculiarities on the seismic site response and its change in contrast to non-porous media. The boundary integral equation method (BIEM) is a well-known computational technique with discussed in the literature advantages for solution of seismic waves in a complex infinite continuum, see Manolis et al. [11]. In what follows, we will present a short state of the art, concerning BIEM for solution of 3D seismic waves in saturated, poro-elastic soils. Generally, the BIEM advantages are as follows: $(a)$ reduction of the model dimensionality; $(b)$ fast solution at selected internal points in terms of boundary-only information and without recourse to domain discretization; $(c)$ high numerical accuracy since quadrature technique is directly applied to the boundary integral equation, which in turn is an exact mathematical statement of the problem under consideration; $(d)$ a priori satisfaction of Sommerfeld's radiation condition at infinity. The BIEM requires two basic ingredients in its formulation, namely a reciprocal relation and a fundamental solution. The fundamental solution plays the key role in describing a given boundary-value problem (BVP) by a system of boundary integral equations (BIE's), based on the reciprocal theorem. The comprehensive state-of-the-art reviews for dynamic fundamental solutions, derived for the porous media and their subsequent incorporation within coupled BIE's can be found in Schanz [12], Gatmiri and Kamalian [13], Gatmiri and Nguyen [14], Seyrafian et al. [15] and Gatmiri and Eslami [16]. Application-type examples, that serve as benchmarks can also be found in the literature, see for instance Theodorakopoulos and Beskos [17], Dominguez [18] and Albers et al. [19]. In Schanz $[8,12]$ various formulations in poroelasticity together with analytical and numerical methods of solutions, associated with these formulations are discussed.

Wave scattering in poroelastic media is investigated by Yamamoto and Kitahara [20], using BIEM. Reflections of plane harmonic waves in a fluid-filled poroelastic half-space are discussed in Lin et al. [21] via analytical approach. Hasheminejad and Avazmohammadi [22] investigated the wave diffraction by two circular cavities in a poroelastic medium. Nenning and Schanz [23] studied wave propagation problems in a poroelastic medium with finite element method (FEM) by introducing infinite 
elements for representing far field behaviour. BIEM results for solution of 3D elastodynamic problems, treating the media as a monophasic pure elastic continuum can be found in Gonsalves et al. [24], Sanchez-Sesma and Luzon [25], Tadeu et al. [26], Galis et al. [27], Chaillat et al. [28], Fu et al. [29], Grasso et al. [30], Chaillat and Bonnet [31], Liu et al. [32], Niu and Dravinski [33].

The available studies on scattering of elastic waves in a three-dimensional poroelastic medium, approximated by a two-phase model are strongly limited and without focus of the site response effects. Related publication are: Zimmerman and Stern [34] studied the scattering of plane P- waves in a poroelastic space with spherical inclusions by BIEM; Zhao and Han [35] investigated the scattering of Rayleigh-waves by a hemi-spherical saturated alluvial valley by Fourier-Bessel series expansion method; Liu et al. [36] investigated the scattering of plane SV waves through spherical inclusions; Cheng and Detournay [37] presented a unified BIEM formulation for the solution of quasi-static, anisotropic poroelasticity; Schanz [8] developed 3-D time domain BEM formulation for wave propagation in poroelastic solids via convolution quadrature method; Ding and Jiang [38], Ding et al. [39] proposed a time domain BIEM for dynamic analysis of saturated porous media, subjected to external forces; Ba et al. [40] studied 3-D scattering of obliquely incident transverse shear SV-waves through an alluvial valley, embedded in a fluid-saturated, poroelastic layered halfspace; Liu et al. [41] presented an indirect BIEM for 3D scattering in a fluid saturated poroelastic half-space.

In a series of papers, Bardet and Morochnik [42-43] proposed an equivalent viscoelastic model, describing the dynamic response of saturated poroelastic materials, that obey Biot's theory. The phase velocity and attenuation of the longitudinal and shear waves in soils are described by a viscoelastic isomorphism, related to Biot's material constants in such a way, that the wave numbers of poroelastic model accord complex wave numbers in the viscoelastic model. This approach has been verified by solution of the 3D problem for scattering of plane compression waves by a spherical poroelastic inhomogeneity in [43], where the authors show that viscoelastic isomorphism solutions accord identical to the original poroelastic problem solution within the specific range of parameters examined.

The brief literature review shows that there is still limited number of BIEM results for synthesis of 3D seismograms in saturated, poroelastic soils containing different types of heterogeneities such as layers, cavities, inclusions or free surface relief. This fact motivates the authors to consider the 3D elastodynamic problem, taking into consideration the soil poroelasticity by the usage of Bardet viscoelastic model and the numerical technique of boundary integral equations. This work is a continuation of the previous authors' results to model 2D plane wave scattering problems in inhomogeneous and heterogeneous geological media $[7,10,11]$ via BIEM, now adding 
effects of the soil poroelasticity and three-dimensional nature of the seismic motion.

The paper is structured as follows: Section 2 gives an overview about the computational model, basing on both the viscoelastic isomorphism to Biot's equations of dynamic poroelasticity presented by the Bardet model and the direct BIEM for 3D wave scattering in a heterogeneous poroelastic half-space. Next, Section 3 discusses results from solution of two illustrative numerical examples for an elastic inclusion, embedded in a poroelastic half-space and a canyon along the free surface of a poroelastic half-space, subjected to plane time-harmonic $\mathrm{P}$ wave, both under consideration of modified site responses. Finally, concluding remarks are given in Section 4.

\section{COMPUTATIONAL MODEL}

\subsection{THE VISCOELASTIC ISOMORPHISM TO BIOT'S EQUATIONS OF DYNAMIC PORO-ELASTICITY - BARDET MODEL}

The most widely-used model, describing the propagation of elastic waves in a porous medium has been proposed by Biot [44] for low-frequency regimes, considered here and by Biot [45] for high-frequencies. The representative elementary volume (REV) of a solid-fluid system with volume $V$ consists of an isotropic elastic porous skeleton with porosity $n$ defined as $n=V_{\text {pore }} / V$, where $V_{\text {pore }}$ is the pore volume. The linear Biot's model in the frame of continual theory is valid if the wavelength $\tilde{\lambda}$ is large in comparison with the characteristic radius of the pores $r$. In low-frequency regime, the Biot's model can be used, when $\tilde{\lambda} \gg L \gg r$, while at high frequencies the Biot's model is correct, when $\tilde{\lambda} \approx L \gg r$, where $L$ is the characteristic size of the REV. The used terminology for poroelastic materials is as follows:

- Solid phase (grains, solid material structure) with the following notations for the elastic bulk module $K_{g}$ and the density $\rho_{g}$.

- Fluid with the following notations for the bulk module $K_{f}$ and the density of fluid $\rho_{f}$.

- Dry porous solid structure (all pores are filled with gas phase/air) with the following material properties: elastic bulk module $K_{\text {dry }}$ and density $\rho_{\text {dry }}$, defined as $\rho_{\text {dry }}=(1-n) \rho_{g}$. The solid-fluid system density is defined as $\rho_{\text {sat }}=$ $\rho_{\text {dry }}+n \rho_{f}=(1-n) \rho_{g}+n \rho_{f}$.

Biot's equations of motion in the case of time-harmonic excitation with frequency $\omega$ in respect to solid displacements $u_{i}(i=x, y, z)$ and fluid displacements $U_{i}$ are as follows:

$$
\begin{gathered}
\mu \Delta u_{i}+\left[\left(\lambda+\mu+Q^{2} / R\right) e_{, i}^{S}+Q \varepsilon_{, i}^{f}\right]+\omega^{2}\left(\rho_{11} u_{i}+\rho_{12} U_{i}\right)=0, \\
\left(Q e_{, i}^{S}+R \varepsilon_{, i}^{f}\right)+\omega^{2}\left(\rho_{21} u_{i}+\rho_{22} U_{i}\right)=0 .
\end{gathered}
$$


Here: the dynamic mass Biot's coefficients are $\rho_{11}=(1-n) \rho_{g}+n\left(\tau_{\alpha}-1\right) \rho_{f}$, $\rho_{12}=\rho_{21}=-n\left(\tau_{\alpha}-1\right) \rho_{f}, \rho_{22}=n \tau_{\alpha} \rho_{f}, \tau_{\alpha}=1+\tau_{r}(1-n) / n, \tau_{r}=0.5$ for sphere, whereas the range of $\tau_{r}$ for other ellipsoids is between 0 and 1, see Mavko et al. [46]; $\Delta$ is the Laplacian operator; $e^{s}=e_{i i}^{s}=u_{i, i}$ and $\varepsilon^{f}=\varepsilon_{i i}^{f}=U_{i, i}, \lambda, \mu$ are Lame constants. It is stated that summation on repeating indices and comma denotes partial differentiation. The shear stiffness of the porous material is captured by the solid skeleton and is not affected by fluid saturation. Due to this fact, both dry and saturated materials have the same shear modulus, $\mu_{\mathrm{dry}}=\mu_{\text {sat }}$ and hitherto denoted by just $\mu$. The Biot's elastic constants $Q, R$, and $P$ are as follows:

$$
\begin{aligned}
& Q=\frac{n K_{g}\left(1-n-\frac{K_{\mathrm{dry}}}{K_{g}}\right)}{\left(1-n-\frac{K_{d r y}}{K_{g}}+n \frac{K_{g}}{K_{f}}\right)} ; \\
& R=\frac{K_{g} n^{2}}{\left(1-n-\frac{K_{d r y}}{K_{g}}+n \frac{K_{g}}{K_{f}}\right)} ; \\
& P=\frac{3\left(1-\nu_{\text {dry }}\right)}{1+\nu_{\text {dry }}} K_{\text {dry }}+\frac{Q^{2}}{R} .
\end{aligned}
$$

The solution for a plane time-harmonic wave, satisfying the Biot's equation of motion was obtained in Bardet [43] and Lin et al. [21]. Following the Biot's wave equation, three mathematical solutions have been identified, corresponding to shear wave $\mathrm{S}$, transmitted through the solid skeleton, the first fast dilatational $\mathrm{P}$ wave and second slow dilatational $\mathrm{P}$ wave. The corresponding wave phase velocities are complex and frequency dependent, hence they correspond to dissipative and dispersive waves. In a series of publications, Bardet [43] discussed the applicability of the viscoelastic behaviour equivalent to Biot's model [44] of dynamic poroelasticity in the low frequency range. As a matter of fact, Bardet [43] proposed a single-phase viscoelastic material representation for saturated, poroelastic soils. At first, the viscoelastic material constants are complex-valued with the real and imaginary part, expressed via Biot's coefficients $Q, R, P$, based on the viscoelastic isomorphism. Therefore, the model proposed by Bardet [43] describes the dissipative wave behaviour with a visco-elastic Kelvin-Voigt model. The following expressions describe the complex-valued phase velocities of visco-elastic materials, following the equivalence between the dynamic responses of the poro-elastic and visco-elastic solids of Kelvin-Voigt rheological model.

For a Kelvin-Voigt model, the complex wave numbers are defined as

$$
k_{S}^{2}=\frac{\omega^{2}}{\left(C_{S}^{*}\right)^{2}}, \quad k_{P}^{2}=\frac{\omega^{2}}{\left(C_{P}^{*}\right)^{2}},
$$


where the complex-valued phase velocities $C_{S}^{*}$ and $C_{P}^{*}$ are

$$
\left(C_{S}^{*}\right)^{2}=C_{S}^{2}\left(1-i \omega \xi_{S}\right) \quad \text { and } \quad\left(C_{P}^{*}\right)^{2}=C_{P}^{2}\left(1-i \omega \xi_{P}\right) .
$$

Here: $C_{S}^{2}=\mu / \rho$ and $C_{P}^{2}=(\lambda+2 \mu) / \rho$ are the real parts of the elastic P-and S-wave velocities, $\xi_{S}$ and $\xi_{P}$ are the corresponding attenuation coefficients, that represent the small hysteretic damping ratios for $\mathrm{P}$ - and $\mathrm{S}$-wave velocities in geomaterial, $\lambda$ and $\mu$ are the real parts of the complex valued Lame constants, $i$ is the imaginary unit. In the low frequency range when $\omega \xi_{P} \ll 1$ and $\omega \xi_{S} \ll 1$, Eqn. (3) can be approximated as

$$
k_{S} \approx \omega\left(1+i 0.5 \omega \xi_{S}\right) / C_{S} \text { and } k_{P} \approx \omega\left(1+i 0.5 \omega \xi_{P}\right) / C_{P} .
$$

Bardet (1992) defines the equivalent effective phase velocities and the corresponding attenuation coefficients, as follows:

$$
\begin{array}{ll}
C_{P}=\sqrt{(P+2 Q+R) / \rho_{\mathrm{sat}}} ; & C_{S}=\sqrt{\mu / \rho_{\mathrm{sat}}}, \\
\xi_{P}=\frac{\rho_{\mathrm{sat}}}{b}\left(\frac{Q+R}{P+2 Q+R} \frac{n \rho_{f}}{\rho_{\mathrm{sat}}}\right)^{2} ; & \xi_{S}=\frac{\rho_{\mathrm{sat}}}{b}\left(\frac{n \rho_{f}}{\rho_{\mathrm{sat}}}\right)^{2},
\end{array}
$$

$b=n^{2} g \rho_{f} / \hat{k}, g$ is the gravity acceleration; $\hat{k}$ is the hydraulic conductivity.

$$
\lambda_{\mathrm{sat}}=\lambda_{\mathrm{dry}}+\frac{Q^{2}}{R}=\frac{3 \nu_{\mathrm{dry}}}{1+\nu_{\mathrm{dry}}} K_{\mathrm{dry}}+\frac{Q^{2}}{R} ; \quad \mu_{\mathrm{sat}}=\mu_{\mathrm{dry}}=\mu
$$

Morochnik and Bardet [42] obtained the approximated Eqns. (6)-(8) in the case of $\omega \rho_{\text {sat }} / b \ll 1$. Due to the fact, that the hydraulic conductivity $\hat{k}$ for soils has small values (for example for sand $\hat{k}=10^{-6} \div 10^{-4}$ ), this condition is fulfilled for lower frequencies, more specifically for the frequencies encountered in earthquake engineering problems. Eqns. (6)-(8) show that the equivalent viscous model considers the soil compression stiffening (bulk modulus), associated with the induced pore pressure during the seismic loading, which causes an increase of $\lambda_{\text {sat }}$ in comparison with $\lambda_{\text {dry }}$, hence increases the value of the longitudinal wave phase velocity in case of saturated soils.

The applicability of Bardet [43] viscoelastic isomorphism to the solution of seismic wave propagation problems in a fluid-saturated half-space is discussed in Dineva et al. [47]. The solutions for free-field motion in a homogeneous poroelastic halfspace under incident longitudinal P-wave, obtained by the two-phase Biot's model in Lin et al. [21] and those obtained by the one-phase viscoelastic Bardet model are identical for low frequencies, see Dineva et al. [47]. The limitations of the Bardet 
model are: (a) it is valid for the low frequency diapason, discussed above; (b) it cannot account for the second (slow) longitudinal wave, because it describes a one-phase viscoelastic material; (c) it is not possible to account for the boundary conditions of pore fluid pressure, since one-phase viscoelastic material is considered. Bardet model shares the same range of applicability dictated by Biot's theory, because it is recovered from the original Biot's model. Regardless of the above mentioned limitations, the main advantage of the Bardet's model is that it can be easily incorporated in the existing BIEM software, avoiding the complicated fundamental solution of the governing equation in Biot's model.

Figure 1 shows the sensitivity of the P-and S-wave velocity for dry and saturated sandstone to the porosity. Results are plotted for values of Poisson's ratio, ranging
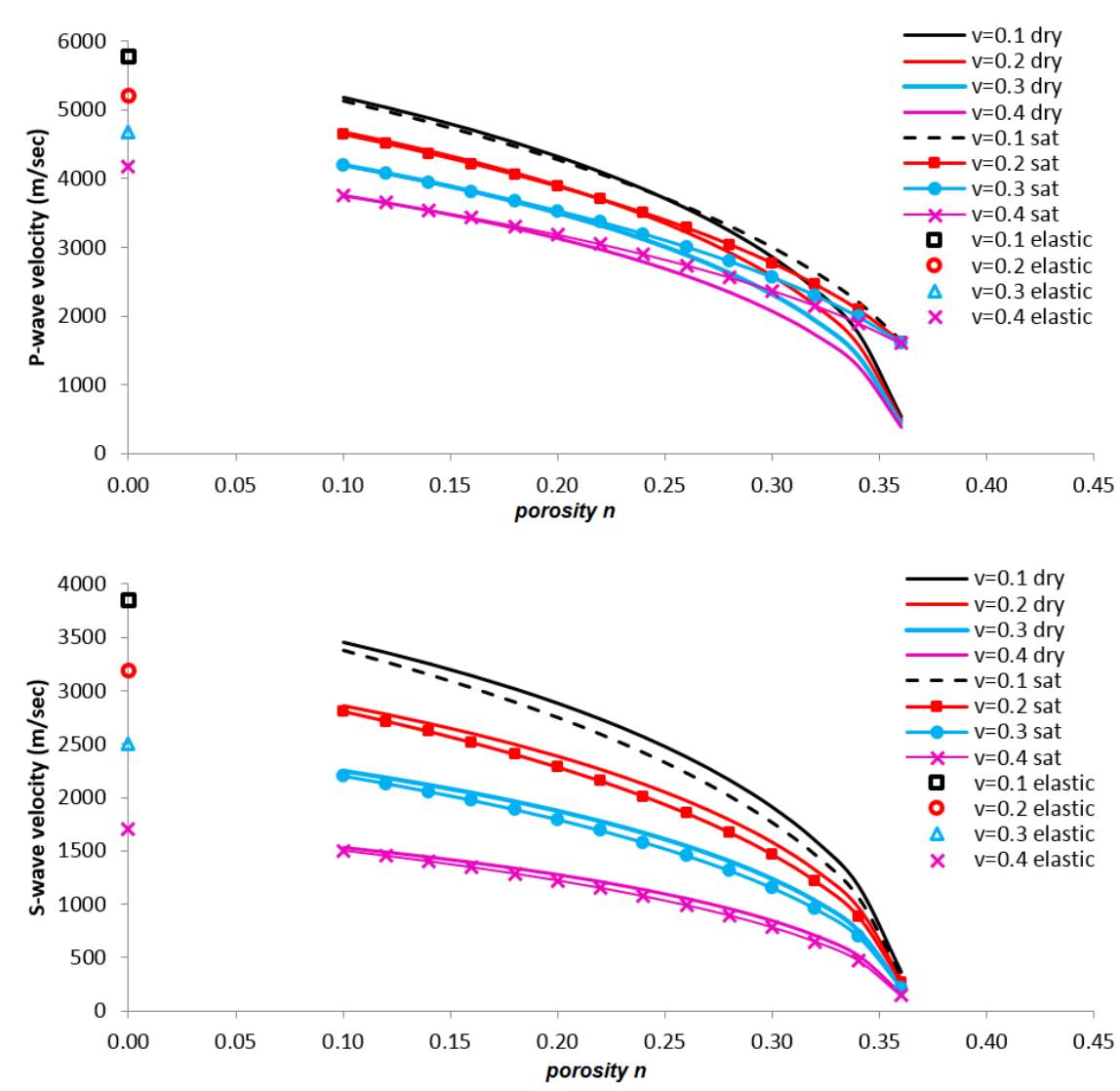

Fig. 1. P-and SV-wave velocity variation with porosity for pure elastic, dry and saturated soil by Bardet (1992) model. 
between 0.1 and 0.4 . For the illustration are used the reference geological material properties, given in Lin et al. [21]: $K_{g}=36000 \mathrm{MPa} ; \rho_{g}=2650 \mathrm{~kg} / \mathrm{m}^{3} ; K_{f}=$ $2000 \mathrm{MPa} ; \rho_{f}=1000 \mathrm{~kg} / \mathrm{m}^{3}$. The dry bulk modulus can be calculated from the relationship, derived from experimental data for poroelstic standstone, given in Gal. et al. (1998) [48] and discussed in Lin et al. (2005): $K_{\mathrm{dry}}=K_{\mathrm{cr}}+\left(1-n / n_{\mathrm{cr}}\right)\left(K_{g}-\right.$ $K_{\mathrm{cr}}$ ), where $K_{\mathrm{cr}}=200 \mathrm{MPa}$ and $n_{\mathrm{cr}}=0.36$. The bulk modulus for a dry porous material is equal to zero at a 'critical' porosity, in which the percentage of pore space in a unit volume becomes too large to form a sustainable solid frame.

As can be seen from Fig. 1, the wave velocity decreases with increasing the porosity. This behaviour is more pronounced in the case of P-waves, for which the wave velocity can express $89 \%$ decrease, comparing with the velocity for small porosity values. Observe that the difference between the P-wave velocity for saturated and dry soil is small for low values of porosity. However, this is no longer true for high values of porosity $(\mathrm{n}>0.3)$, where the wave velocity of saturated and dry soil can differ significantly up to $67 \%$. For dry materials and for low porosity value, the results are nearly the pure elastic case. Note also, that the wave velocity decreases by increasing the Poisson's ratio values, whereas this trend is eliminated by increasing the porosity.

\subsection{PROBLEM FORMULATION: GOVERNING EQUATION AND BOUNDARY CON- DITIONS}

Consider in Fig. 2, a 3D finite local geological heterogeneity $\Omega_{1}$, such as a canyon or an elastic inclusion with arbitrary geometry and the following material properties: density $\rho_{1}$, Lame constants $\lambda_{1}$ and $\mu_{1}$, phase velocities $C_{p}^{\left(\Omega_{1}\right)}=\sqrt{\left(\lambda_{1}+2 \mu_{1}\right) / \rho_{1}}$ and $C_{S}^{\left(\Omega_{1}\right)}=\sqrt{\mu_{1} / \rho_{1}}$. The boundary of the inclusion is denoted by $\Gamma_{\Omega_{1}}=\Gamma \cup \Gamma_{\mathrm{ff}}$, where $\Gamma_{\mathrm{ff}}$ is the free-surface of the heterogeneity and $\Gamma$ is the interface between $\Omega_{1}$ and $\Omega_{0}$ (the dotted line in Fig. 2). The finite geological heterogeneity $\Omega_{1}$ is situated in a homogeneous poro-elastic half-space $\Omega_{0}$ with material characteristics porosity $n$, phase velocities $C_{p}^{\left(\Omega_{0}\right)}, C_{S}^{\left(\Omega_{0}\right)}$ and the corresponding attenuation coefficients $\xi_{P}^{\left(\Omega_{0}\right)}$, $\xi_{S}^{\left(\Omega_{0}\right)}$, computed by expressions (6) and (7). An incident time-harmonic plane P/SVwave with frequency $\omega$ impinges on the heterogeneity. Inside the range of $\Omega_{1}$ (when consider elastic inclusion) the scattered wave field $u_{i}^{\text {sc }}, t_{i}^{\text {sc }}$ is equal to the total wave field $u_{i}, t_{i}$. Outside the range of $\Omega_{1}$, the total wave field $u_{i}, t_{i}$ is equal to the sum of the scattered by the heterogeneity $\Omega_{1}$ wave field $u_{i}^{\mathrm{sc}}, t_{i}^{\mathrm{sc}}$ and the free field wave motion $u_{i}^{\mathrm{ff}}, t_{i}^{\mathrm{ff}}$ in $\Omega_{0}$. The free field wave motion is defined as the wave field in the half-space without any heterogeneity. The solution for the free field wave motion can be found in Achenbach [49] and Omidvar et al. [50]. The expressions for the displacement and traction free-field motion are given in the Appendix. The governing 


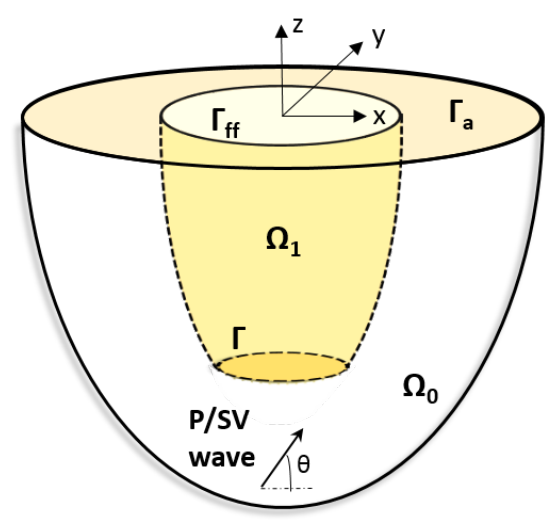

Fig. 2. Scattering of plane time-harmonic wave by a 3D heterogeneity in a poroelastic halfspace.

equation in frequency domain for $l=1$ or $l=0$ is

$$
\left(\left(C_{P}^{\Omega_{l}}\right)^{2}-\left(C_{S}^{\Omega_{l}}\right)^{2}\right) u_{i, i j}+\left(C_{P}^{\Omega_{l}}\right)^{2} u_{j, i i}+\omega^{2} u_{j}=0 \quad i, j=x, y, z .
$$

Note, that in the case of $l=0$, the phase velocities of longitudinal and shear waves are complex-valued, due to the viscoelastic behaviour of the saturated soil.

The boundary conditions are as follows:

- at $z=0$; along the free surface, tractions are equal to zero

$$
t_{k}=0 \quad \text { for } k=x, y, z,
$$

- along the rest of the heterogeneity's sides (when it is elastic inclusion), i.e. along the interface boundary $(x, y, z) \in \Gamma$, between the elastic inclusion $\Omega_{1}$ and the half-space $\Omega_{0}$, the following conditions should be satisfied:

$$
\begin{aligned}
& u_{k}^{\left(\Omega_{1}\right)}(x, y, z, \omega)=u_{k}^{\mathrm{ff},\left(\Omega_{0}\right)}(x, y, z, \omega)+u_{k}^{\mathrm{sc},\left(\Omega_{0}\right)}(x, y, z, \omega) \\
& \text { outside } \Omega_{1} \\
& t_{k}^{\left(\Omega_{1}\right)} \underset{\text { inside } \Omega_{1}}{(x, y, z, \omega)}=-\left[t_{i}^{\mathrm{ff},\left(\Omega_{0}\right)}(x, y, z, \omega)+t_{i}^{\mathrm{sc}, \Omega_{0}}(x, y, z, \omega)\right] . \\
& \text { outside } \Omega_{1}
\end{aligned}
$$

Finally, in the semi-infinite half-space $\Omega_{0}$, the Sommerfeld's radiation condition is satisfied at infinite. 


\subsection{DiRECT BIEM FOR 3D WAVE SCATTERING IN A POROELASTIC HALF-SPACE}

The above boundary-value problem (BVP) is here reformulated via boundary integral equations (BIE's) using Betti's reciprocal theorem in conjunction with the frequency dependent fundamental solution for 3D wave motion, see Dominguez [18]. What follows is a presentation of the BIEM formulation of the formulated in Section 2.2 boundary-value problems in case that the local 3D heterogeneity is an elastic inclusion (for example, alluvial basin/foundation) or a free surface canyon.

\section{LOCAL SITE HETEROGENEITY IN FORM OF AN ELASTIC INCLUSION}

The elastic inclusion is situated in $\Omega_{1}$ with boundaries $\Gamma_{\Omega_{1}}$. The following boundary integral equation holds for the external domain with boundary $\Gamma_{\text {ext }}=\Gamma \cup \Gamma_{a}$, where $\Gamma_{a}$ is the free surface in $\Omega_{0}$ outside $\Gamma_{\mathrm{ff}}$

$$
\begin{array}{r}
c_{i j}\left(u_{j}(\boldsymbol{\chi}, \omega)-u_{j}^{\mathrm{ff}}(\boldsymbol{\chi}, \omega)\right)=\int_{\Gamma_{\mathrm{ext}}} U_{i j}^{*\left(\Omega_{0}\right)}(\boldsymbol{\chi}, \boldsymbol{\xi}, \omega)\left(t_{j}(\boldsymbol{\xi}, \omega)-t_{j}^{\mathrm{ff}}(\boldsymbol{\xi}, \omega)\right) d \Gamma_{\mathrm{ext}}(\boldsymbol{\xi}) \\
-\int_{\Gamma_{\mathrm{ext}}} P_{i j}^{*\left(\Omega_{0}\right)}(\boldsymbol{\chi}, \boldsymbol{\xi}, \omega)\left(u_{j}(\boldsymbol{\xi}, \omega)-u_{j}^{\mathrm{ff}}(\boldsymbol{\xi}, \omega)\right) d \Gamma_{\mathrm{ext}}(\boldsymbol{\xi}) ; \quad \chi \in \Gamma_{\mathrm{ext}} .
\end{array}
$$

Inside the range of elastic inclusion $\Omega_{1}$ with boundary $\Gamma_{\Omega_{1}}=\Gamma \cup \Gamma_{\mathrm{ff}}$, the following boundary integral equation holds:

$$
\begin{aligned}
c_{i j} u_{j}(\boldsymbol{\chi},)=\int_{\Gamma_{\Omega_{1}}} U_{i j}^{*\left(\Omega_{1}\right)}(\boldsymbol{\chi}, \boldsymbol{\xi}, \omega) t_{j}(\boldsymbol{\xi}, \omega) d \Gamma_{\Omega_{1}}(\boldsymbol{\xi}) \\
\quad-\int_{\Gamma_{\Omega_{1}}} P_{i j}^{*\left(\Omega_{1}\right)}(\boldsymbol{\chi}, \boldsymbol{\xi}, \omega) u_{j}(\boldsymbol{\xi}, \omega) d \Gamma_{\Omega_{1}}(\boldsymbol{\xi}) ; \quad \boldsymbol{\xi} \in \Gamma_{\Omega_{1}} .
\end{aligned}
$$

where: $\chi$ and $\boldsymbol{\xi}$ are the position vectors of the source and field point, respectively; $c_{i j}$ is the jump term depending on the surface geometry at the collocation point; $U_{i j}^{*\left(\Omega_{0}\right)}$, $U_{i j}^{*\left(\Omega_{1}\right)}$ is the fundamental solution for displacement in domains $\Omega_{0}$ and $\Omega_{1}$, respectively; $P_{i j}^{*}(\boldsymbol{\chi}, \boldsymbol{\xi}, \omega)=\sigma_{i j k}^{*}(\boldsymbol{\chi}, \boldsymbol{\xi}, \omega) n_{k}(\boldsymbol{\xi})$ is the corresponding traction fundamental solution; $\sigma_{i j k}^{*}=C_{i j m n} \partial U_{m k}^{*} / \partial \xi_{n}=-C_{i j m n} \partial U_{m k}^{*} / \partial \chi_{n}, n_{k}$ are the components of the outward pointing unit normal vector; $C_{i j k l}$ is the stiffness tensor. The expression for the fundamental solution can be found in Dominguez [18]. The unknowns in the system of BIE's (12), (13) are the total displacement along free surface $(z=0)$, and the total wave field (displacement and traction) along the interface surface $\Gamma$. The numerical realization of this problem, however, is in respect to the scattered wave 
field, thus leading to higher accuracy, due to the well-known fact that the scattered wave field satisfies the Sommerfeld's radiation condition at infinite. Here, we will describe shortly the discretization procedure following Reinoso et al. [51]. After discretization of the boundary integral equations for the external zone with boundary $\Gamma_{a} \cup \Gamma$, (outside the inclusion), (see, Fig. 2), the following matrix form of the algebraic equations with respect to the scattered displacement vector $\boldsymbol{u}_{s}=\boldsymbol{u}_{s}^{\alpha}+\boldsymbol{u}_{s}^{\Gamma}$ and traction vector $\boldsymbol{t}_{s}=\boldsymbol{t}_{s}^{\alpha}+\boldsymbol{t}_{s}^{\Gamma}$ is obtained

$$
\boldsymbol{H}_{0} \boldsymbol{u}_{s}-\boldsymbol{G}_{0} \boldsymbol{t}_{s}=\mathbf{0} \Rightarrow \boldsymbol{H}_{0}\left(\boldsymbol{u}_{s}^{\alpha}+\boldsymbol{u}_{s}^{\Gamma}\right)-\boldsymbol{G}_{0}\left(\boldsymbol{t}_{s}^{\alpha}+\boldsymbol{t}_{s}^{\Gamma}\right)=0 .
$$

Here, $\boldsymbol{u}_{s}^{\alpha}, \boldsymbol{u}_{s}^{\Gamma}, \boldsymbol{t}_{s}^{\alpha}, \boldsymbol{t}_{s}^{\Gamma}$ are scattered displacement and traction vectors along boundaries $\Gamma_{a}$ and $\Gamma, \boldsymbol{H}_{0}, \boldsymbol{G}_{0}$ are the corresponding influence matrices in $\Omega_{0}$ along $\Gamma_{a} \cup \Gamma$. For the total traction vector along boundary $\Gamma_{a}$ can be written

$$
\underset{\text { total }}{\boldsymbol{t}^{\alpha}}=\underset{\text { scattered }}{\boldsymbol{t}_{s}^{\alpha}}+\boldsymbol{t}_{\mathrm{ff}}^{\alpha}=0 \Rightarrow \underset{\text { scattered }}{\boldsymbol{t}_{s}^{\alpha}}=-\boldsymbol{t}_{\mathrm{ff}}^{\alpha} .
$$

Having in mind Eqn. (15), the matrix equation Eqn. (14) can be written in the form

$$
\boldsymbol{H}_{0}\left(\boldsymbol{u}_{s}^{\alpha}+\boldsymbol{u}_{s}^{\Gamma}\right)-\boldsymbol{G}_{0} \boldsymbol{t}_{s}^{\Gamma}=\boldsymbol{G}_{0} \boldsymbol{t}_{\mathrm{ff}}^{\Gamma} ; \quad \text { in } \Omega_{0} .
$$

Note, that the suffix "ff" in the field variables stands for the free field motion, while the suffix "s" for the scattered wave field.

After discretization of the boundary integral equation for the internal zone $\Omega_{1}$ with boundary $\Gamma_{\mathrm{ff}} \cup \Gamma$, (inside the inclusion), (see, Fig. 2), the matrix form of the algebraic equations with respect to the scattered (equals to the total) displacement and traction vector is obtained in Eqn. (17), having in mind, that $\boldsymbol{u}_{1}^{\Gamma}=\boldsymbol{u}_{0}^{\Gamma}-\boldsymbol{u}_{\mathrm{ff}}^{\Gamma}$ and $\boldsymbol{t}_{1}^{\Gamma}=-\boldsymbol{t}_{0}^{\Gamma}=-\boldsymbol{t}_{s}^{\Gamma}-\boldsymbol{t}_{\mathrm{ff}}^{\Gamma}$ along $\Gamma$.

$$
\begin{aligned}
& \boldsymbol{H}_{1}\left[\boldsymbol{u}_{1}^{\mathrm{ff}}+\boldsymbol{u}_{1}^{\Gamma}\right]-\boldsymbol{G}_{1}\left[\boldsymbol{t}_{1}^{\mathrm{ff}}+\boldsymbol{t}_{1}^{\Gamma}\right]=0 \\
& \boldsymbol{H}_{1}\left[\boldsymbol{u}_{1}^{\mathrm{ff}}+\boldsymbol{u}_{s}^{\Gamma}+\boldsymbol{u}_{\mathrm{ff}}^{\Gamma}\right]-\boldsymbol{G}_{1}\left[-\boldsymbol{t}_{\mathrm{ff}}^{\Gamma}-\boldsymbol{t}_{s}^{\Gamma}\right]=0 \quad \text { in } \Omega_{1} \\
& \boldsymbol{H}_{1} \boldsymbol{u}_{1}^{\mathrm{ff}}+\boldsymbol{H}_{1} \boldsymbol{u}_{s}^{\Gamma}+\boldsymbol{G}_{1} \boldsymbol{t}_{s}^{\Gamma}=-\boldsymbol{H}_{1} \boldsymbol{u}_{\mathrm{ff}}^{\Gamma}-\boldsymbol{G}_{1} \boldsymbol{t}_{\mathrm{ff}}^{\Gamma}
\end{aligned}
$$

Where: $\boldsymbol{H}_{1}, \boldsymbol{G}_{1}$ are the influence matrices in $\Omega_{1}$ along boundaries $\Gamma_{\mathrm{ff}} \cup \Gamma ; \boldsymbol{u}_{1}^{\mathrm{ff}}, \boldsymbol{t}_{1}^{\mathrm{ff}}$ are the displacement and traction vector, correspondingly, inside $\Omega_{1}$ along boundary $\Gamma_{\mathrm{ff}}$.

Equation (16) for the external zone $\Omega_{0}$ and Eq. (17) for the internal zone $\Omega_{1}$ form the following system (18) of linear algebraic equations $A X=B$ :

$$
\left[\begin{array}{cccc}
\boldsymbol{H}_{0} & \boldsymbol{H}_{1} & \mathbf{0} & -\boldsymbol{G}_{0} \\
& \boldsymbol{H}_{1} & \boldsymbol{H}_{1} & \boldsymbol{H}_{1}
\end{array}\right]\left[\begin{array}{c}
\boldsymbol{u}_{s}^{a} \\
\boldsymbol{u}_{s}^{\Gamma} \\
\boldsymbol{u}_{1}^{\mathrm{ff}} \\
\boldsymbol{t}_{s}^{\Gamma}
\end{array}\right]=\left(-\boldsymbol{G}_{0} \boldsymbol{t}_{\mathrm{ff}}^{a}-\boldsymbol{H}_{1} \boldsymbol{u}_{\mathrm{ff}}^{\Gamma}-\boldsymbol{G}_{1} \boldsymbol{t}_{\mathrm{ff}}^{\Gamma}\right)
$$


The unknowns are scattered displacement vectors $\boldsymbol{u}_{1}^{\mathrm{ff}}, \boldsymbol{u}_{s}^{\Gamma}, \boldsymbol{u}_{s}^{a}$ along boundaries $\Gamma_{\mathrm{ff}}$, $\Gamma, \Gamma_{a}$, correspondingly and scattered traction vector $\boldsymbol{t}_{s}^{\Gamma}$ along boundary $\Gamma$, while $\boldsymbol{u}_{\mathrm{ff}}^{\Gamma}$ and $t_{\mathrm{ff}}^{\Gamma}$ are the known vectors of displacement and traction for the free field motion along $\Gamma$, presented in the Appendix.

\section{LOCAL SITE HETEROGENEITY IN FORM OF A CANYON}

In this case, the following boundary integral equation along the surface $\Gamma_{\text {canyon }}=$ $\Gamma \cup \Gamma_{a}$ (see Fig. 2) describes the problem

$$
\begin{aligned}
c_{i j}\left(u_{j}(\boldsymbol{\chi}, \omega)-u_{j}^{\mathrm{ff}}(\boldsymbol{\chi}, \omega)\right) & =\int_{\Gamma_{\text {canyon }}} U_{i j}^{*}(\boldsymbol{\chi}, \boldsymbol{\xi}, \omega)\left(t_{j}(\boldsymbol{\xi}, \omega)-t_{j}^{\mathrm{ff}}(\boldsymbol{\xi}, \omega)\right) d S(\boldsymbol{\xi}) \\
& -\int_{\Gamma_{\text {canyon }}} P_{i j}^{*}(\boldsymbol{\chi}, \boldsymbol{\xi}, \omega)\left(u_{j}(\boldsymbol{\xi}, \omega)-u_{j}^{\mathrm{ff}}(\boldsymbol{\xi}, \omega)\right) d S(\boldsymbol{\xi})
\end{aligned}
$$

After discretization of BIE (Eqn. 19), the following algebraic system with respect to the total displacement vector $\boldsymbol{u}(3 N \times 1)$ is obtained:

$$
\begin{aligned}
& \boldsymbol{H} \boldsymbol{u}^{\mathrm{sc}}=\boldsymbol{G} \boldsymbol{t}^{\mathrm{sc}} \rightarrow \boldsymbol{H}\left(\boldsymbol{u}-\boldsymbol{u}^{\mathrm{ff}}\right)=\boldsymbol{G}\left(\boldsymbol{t}-\boldsymbol{t}^{\mathrm{ff}}\right) \quad \text { consider } \boldsymbol{t}=0 \\
& \boldsymbol{H}[3 N \times 3 N] \boldsymbol{u}(3 N \times 1)=\boldsymbol{H}[3 N \times 3 N] \boldsymbol{u}^{\mathrm{ff}}(3 N \times 1) \\
&-\boldsymbol{G}[3 N \times 3 N] \boldsymbol{t}^{\mathrm{ff}}(3 N \times 1)
\end{aligned}
$$

The matrices $\boldsymbol{G}$ and $\boldsymbol{H}$ are the final global influence matrices. They are full matrices of size $3 N \times 3 N$, where $N$ is the number of global boundary nodal points.

The numerical solution of both BVPs follows the standard BIEM collocation procedure. In particular, the surface boundary is discretized into quadratic triangle isoperimetric (six-node) boundary elements, using continuous polynomial approximations for the boundary geometry, the displacement and the traction vectors. Two types of integrals are obtained after discretization of BIE, depending on whether or not the radial distance $r$ between the source and receiver points is zero: (a) at $r \neq 0$ the integrals are regular, there are no singularities, solution is numerical; (b) at $r \rightarrow 0$ there are two types of singularities: $(i)$ the displacement-based kernels $\int U_{i j}^{*}(x, \xi, \omega) t_{j}(\xi, \omega) d \xi$ exhibit a weak singularity of type $O(1 / r)$ and these integrals are solved by appropriate quadrature rule explained in Dominguez (1993); (ii) the traction-based kernels $\int P_{i j}^{*}(x, \xi, \omega) u_{j}(\xi, \omega) d \xi$ exhibit a strong singularity of the type $O\left(1 / r^{2}\right)$ and these type of integrals are solved via application of the wellknown rigid body motion concept. After integrating both weak and strong singularities in the integrands, computing all integrals and satisfying the prescribed boundary conditions, an algebraic system of equations is obtained, rearranged and solved with 
respect to the unknown displacements and tractions in terms of the prescribed values, all in the frequency domain. In general, BIEM mesh discretization issues, such as mesh density is controlled by the solution of the following test example, demonstrating the verification of the applied numerical algorithm. In the case of equal material characteristics of the half-space and the elastic inclusion, the solution of the above formulated BVP's accord the free field solution for a 3D wave propagation in an elastic homogenous half-space, subjected to incident time-harmonic wave.

(a)

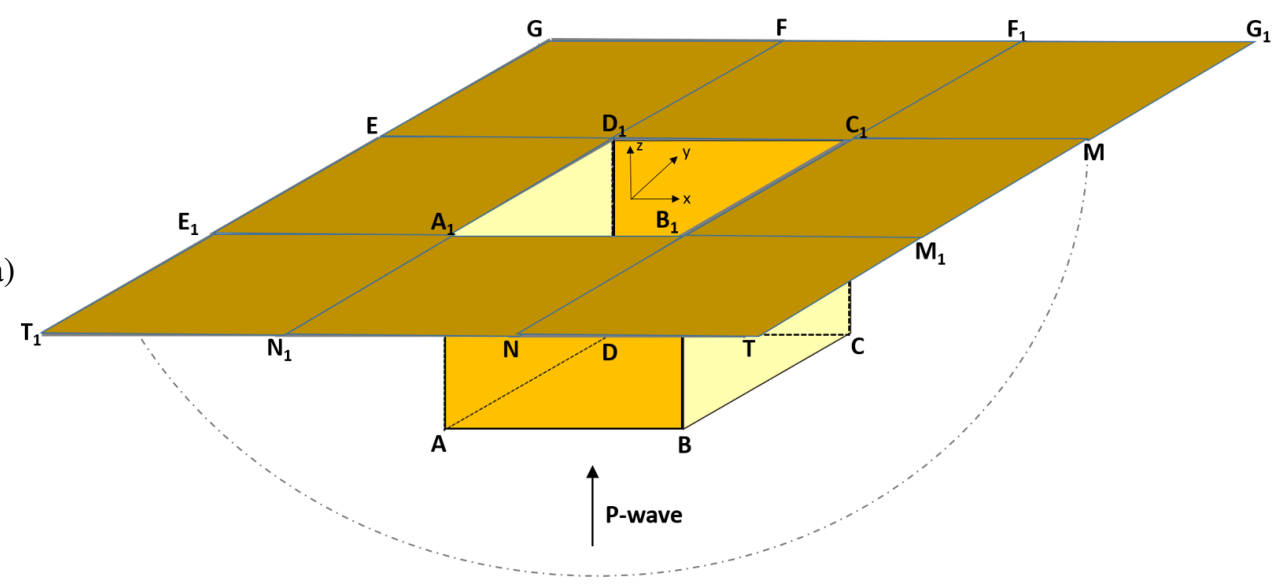

(b)

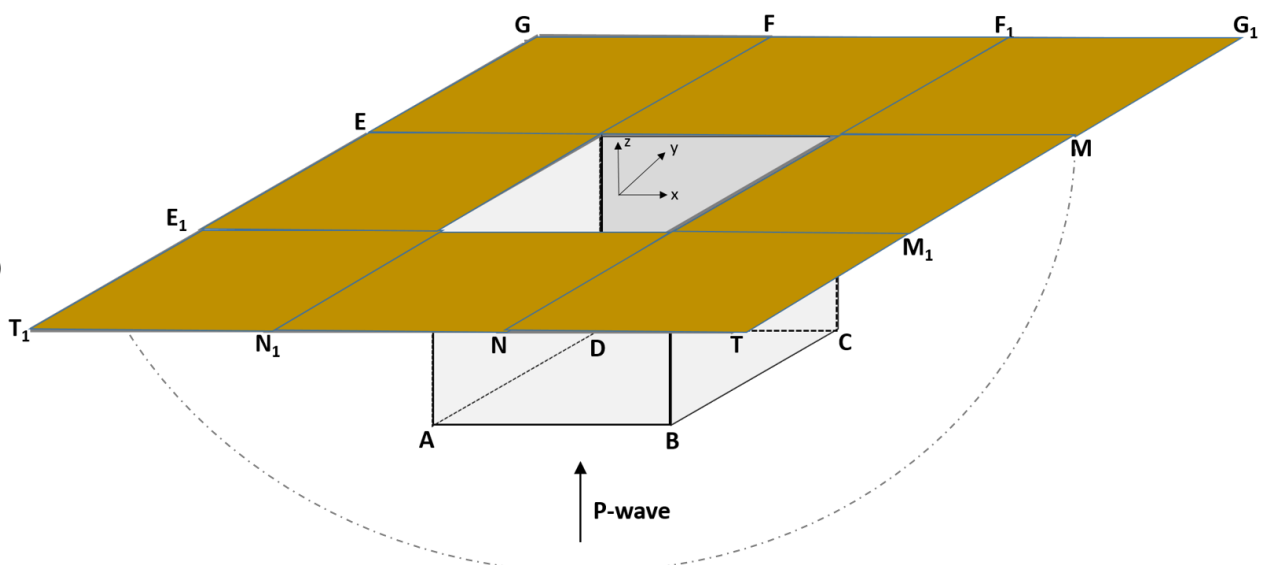

Fig. 3. (a) A pure elastic heterogeneity $A B C D A_{1} B_{1} C_{1} D_{1}$ or (b) a canyon with prism geometry $800 \times 800 \times 800 \mathrm{~m}$, embedded in a poroelastic half-space under incident timeharmonic P-wave. The additional discretization area along the free surface is presented by 8 squares $T_{1} N_{1} E_{1} A_{1}, N_{1} N A_{1} B_{1}, N T B_{1} M_{1}, B_{1} M_{1} C_{1} M, C_{1} F_{1} M G_{1}, C_{1} F_{1} D_{1} F$, $D_{1} F E G, E_{1} E A_{1} D_{1}$, all with size of $800 \mathrm{~m}$. 


\section{NUMERICAL SIMUlations AND SITE RESPONSE STUdiES}

In this section, we apply the foregoing discussed computational scheme to solve the defined two boundary-value problems. All numerical examples, that follow serve to visualize the wave field modification under assumption of porous systems and to demonstrate the potential of the proposed simple visco-elastic mechanical model and accompanied numerical tool to deal with wave propagation in spatial 3D heterogeneous poroelastic geological media. As explained before, the following two types of 3D site heterogeneities are analysed: a canyon and an elastic inclusion.

\section{SITE RESPONSE STUDY - COMBINED EFFECT OF TOPOGRAPHY AND POROELAS- TICITY}

Consider a finite canyon embedded in a poroelastic soil half-space. An incident timeharmonic plane $\mathrm{P}$-wave with frequency $\omega$ is propagating vertically in the plane $x-z$ with incidence angle $\theta=\pi / 2$ according to axis $O x$. The used BIEM mesh consists of 576 triangle quadratic isoperimetric boundary elements, along the canyon's sides

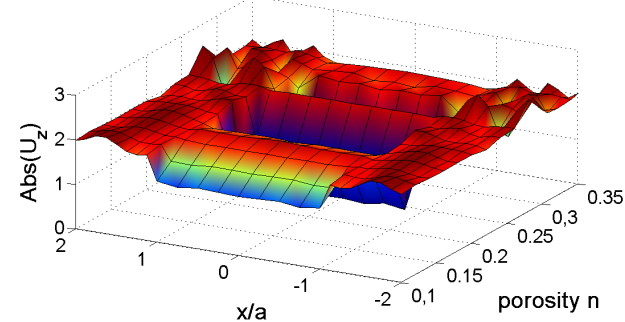

(a)

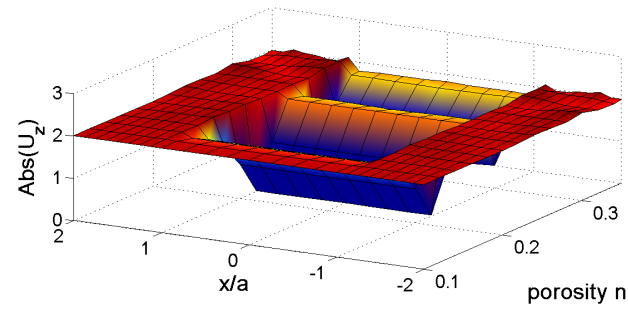

(c)

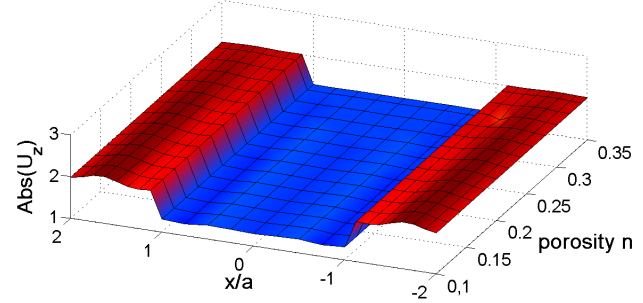

(b)

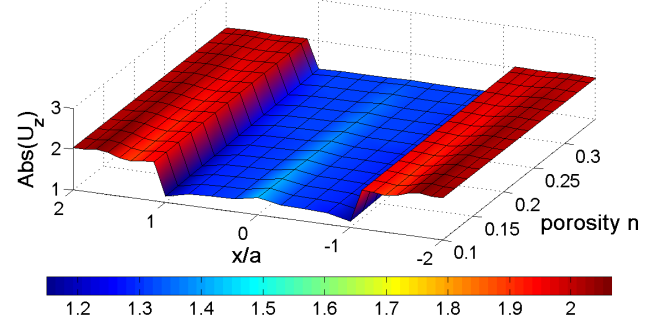

(d)

Fig. 4. Displacement amplitudes $\left|u_{z}\right|$ versus $x / a(a=400 \mathrm{~m})$ along the free surface (at line $y=0$ ) of a poro-elastic half-space, containing a canyon under vertical incident timeharmonic P-wave with frequency $f=6.4 \mathrm{~Hz}$ for: (a) dry soil with Poisson ratio $\nu=0.4$; (b) saturated soil with Poisson ratio $\nu=0.4$; (c) dry soil with Poisson ratio $\nu=0.1$; and (d) saturated soil with Poisson ratio $\nu=0.1$. 


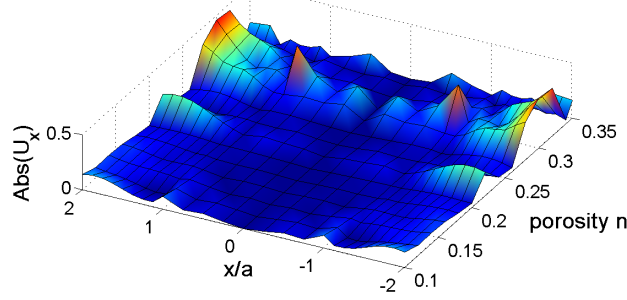

(a)

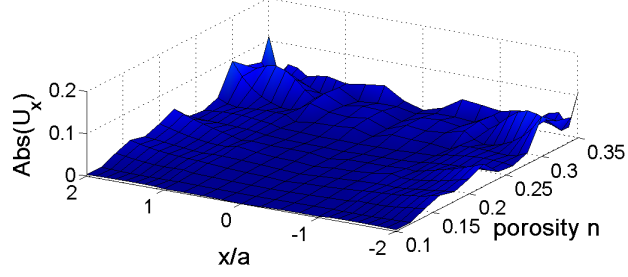

(c)

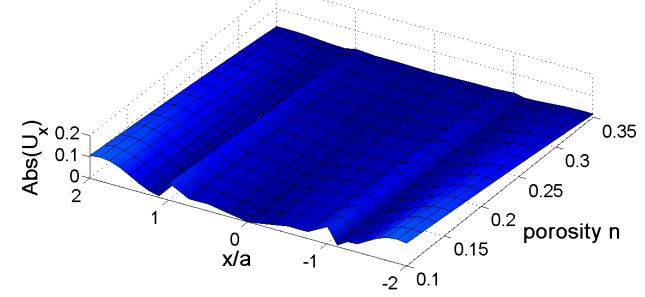

(b)

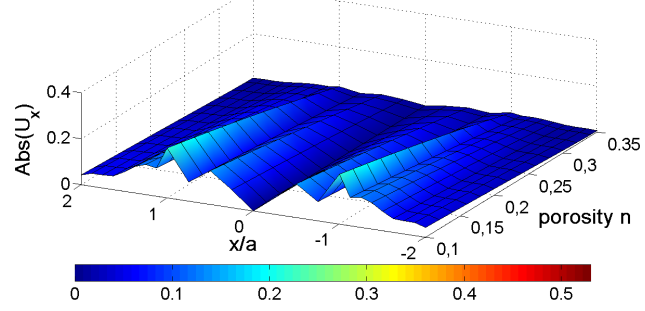

(d)

Fig. 5. Displacement amplitudes $\left|u_{x}\right|$ versus $x / a(a=400 \mathrm{~m})$, along the free surface (at line $y=0$ ) of a poroelastic half-space, containing a canyon under time-harmonic P-wave propagating along axis $O z$ with frequency $f=6.4 \mathrm{~Hz}$ for: (a) dry soil with Poisson ratio $\nu=0.4$; (b) saturated soil with Poisson ratio $\nu=0.4$; (c) dry soil with Poisson ratio $\nu=0.1$; and (d) saturated soil with Poisson ratio $\nu=0.1$.

and along the free surface out of the canyon, respectively. The number of the global boundary nodes is $N=1201$, and the algebraic system after discretisation is of size 3603 (see Fig. 3).

Figures $4 \mathrm{a}, \mathrm{b}, \mathrm{c}, \mathrm{d}$ present the displacement amplitudes $\left|u_{z}\right|$ due to time-harmonic vertically incident $\mathrm{P}$-wave with frequency $6.4 \mathrm{~Hz}$ versus ratio $x / a(a=400 \mathrm{~m})$ along the free surface $z=0$ on the line $y=0$. The Poisson's ratio is $\nu_{\text {dry }}=0.4$ or 0.1 and the porosity is between 0.1 and 0.35 . The obtained results are for dry (Figs. 4a, c) and saturated (Figs. 4b, d) poro-elastic sandstone with Poisson's ratio equal to 0.4 and 0.1. The analogous results for the displacement amplitudes $\left|u_{x}\right|$ are shown in Figs. 5a, b, c, d.

Displacement amplitudes $\left|u_{z}\right|$ along the canyon's bottom $A B C D$ (see Fig. 3) at frequency $f=6.4 \mathrm{~Hz}$ of time-harmonic vertical incident $\mathrm{P}$-wave propagating in poroelastic soil with porosity $n=0.1$ and $n=0.3$ are shown in Figs, 6-7, correspondingly, for the cases of (a) dry and (b) saturated soil. The frequency $f=$ $6.4 \mathrm{~Hz}$ corresponds to the following value of the non-dimensional frequency $\Omega=$ $(\omega a) /\left(\pi C_{S}\right)=(2 a) \lambda_{S}=5$, where $\lambda_{S}$ is the wavelength of the shear wave and $2 a=800 \mathrm{~m}, 2 a$ is the cube's size. 
(a)

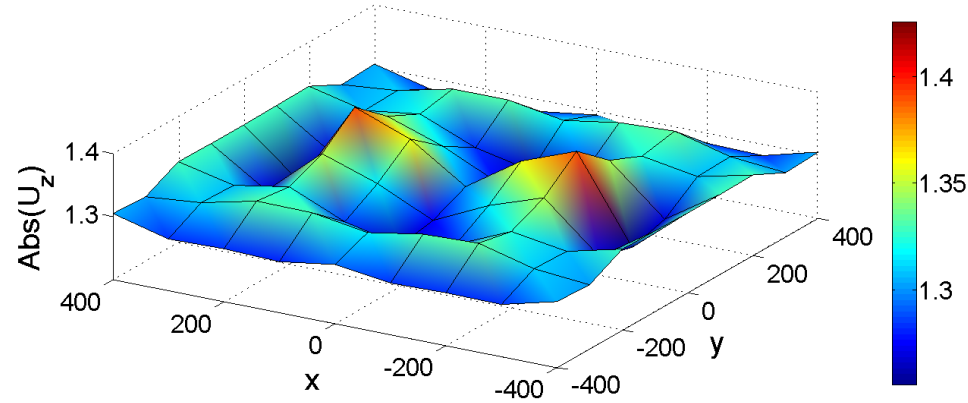

(b)

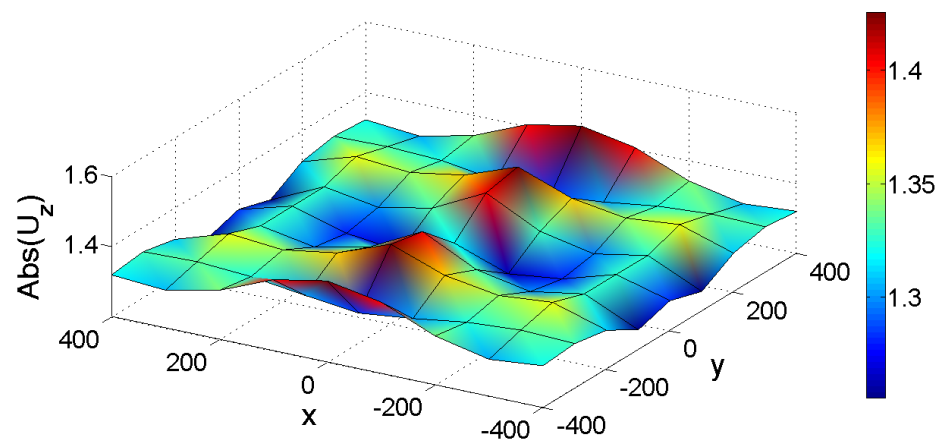

Fig. 6. Displacement amplitude $\left|u_{z}\right|$ along the canyon situated in a poroelastic half-space with porosity $n=0.1$ for: (a) dry; (b) saturated soil material, under time-harmonic P-wave propagating along the axis $O z$ with frequency $f=6.4 \mathrm{~Hz}$.

Figures 4-7 demonstrate the influence of porous media on the seismic signals, computed at the surface of a canyon resting on a poroelastic half-space. The first observation is that for almost all analyzed porosities, the displacement amplitudes of saturated soil are smaller than those for dry soils. This is due to the fact, that the increasing pore pressure induced by the passage of seismic waves tends to stiffen the porous soil/rock skeleton and hence, to reduce the appearing deformation. The shear strength of Biot's porous material is provided by the solid skeleton and it is assumed to be unaffected by the fluid saturation, which implies $\mu_{\text {sat }}=\mu_{\text {dry }}$.

On the other hand, the Lame coefficient $\lambda$ changes to $\lambda_{\text {sat }}=\lambda_{\text {dry }}+Q^{2} / R$, and thus indicates a strengthening of the porous skeleton, due to dilatation deformation, followed by an increase of the phase velocity of longitudinal P-wave. In particular, the P-wave characteristics are modified significantly by the change from dry to saturated geomaterial. Additional damping effect occurs due to the viscous type of the fluid and the matrix behaviour. However, for low porosity value $n=0.1$ there is 
(a)

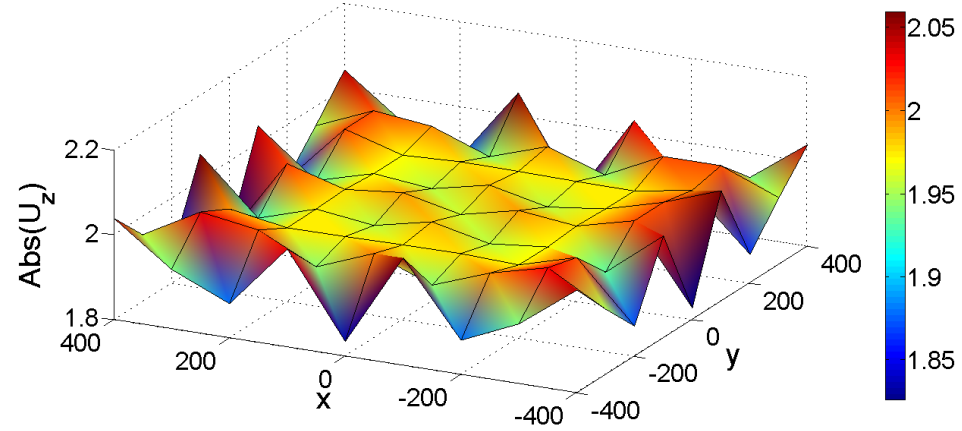

(b)

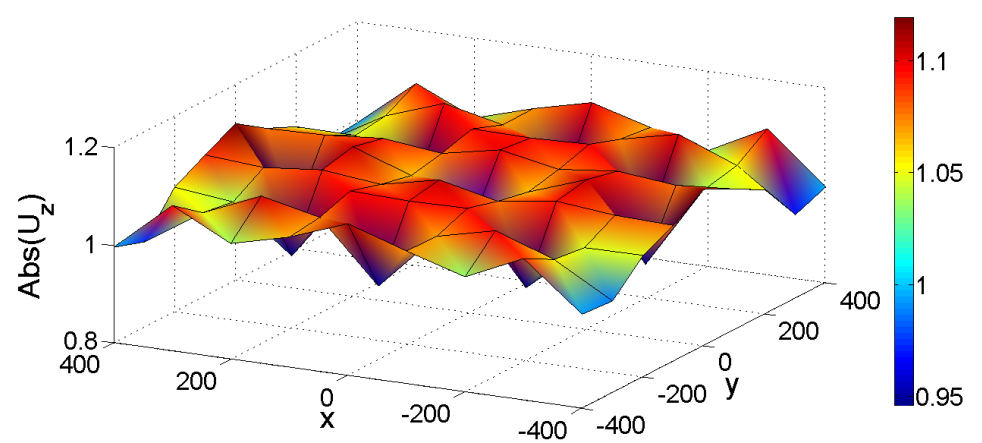

Fig. 7. Displacement amplitude $\left|u_{z}\right|$ along the canyon, situated in a poroelastic half-space with porosity $n=0.3$ for: (a) dry; (b) saturated soil material, under time-harmonic P-wave propagating along the axis $O z$ with frequency $f=6.4 \mathrm{~Hz}$.

almost no difference between the seismic response in case of dry and saturated soil. In this case, the dry frame strength increases and becomes a pure elastic modulus. In consequence, it is obvious that the seismic response changes significantly, when we consider saturated, porous geological media. It must be noticed, that the above conclusions are in agreement with the effects visible in Fig. 1(a).

\section{SITE RESPONSE STUDY - COMBINED EFFECT OF ELASTIC HETEROGENEITY AND POROELASTICITY}

In what follows, we investigate the seismic field in a poroelastic half-space, containing an elastic inclusion. The geometry of the finite elastic inclusion $A B C D A_{1} B_{1} C_{1} D_{1}$ (see Fig. 3) is rectangular with size $10 \times 10 \mathrm{~m}$ along the free surface $(z=0)$ and with depth equal to $3 \mathrm{~m}$. The inclusion is surrounded by the top side $A_{1} B_{1} C_{1} D_{1}$, bottom side $A B C D$ and the following four sides $A_{1} A B_{1} B, B_{1} B C_{1} C, C_{1} C D_{1} D$ and $D_{1} D A_{1} A$ embedded in a poroelastic soil half-space. Note, that for the case of 
the elastic inclusion the top side $A_{1} B_{1} C_{1} D_{1}$ exists compared with the previous case of the canyon. The used BIEM mesh consists of 24 triangle quadratic boundary elements ( 50 global nodes) for the inclusion and 52 triangle quadratic boundary elements (117 global nodes) along the free surface boundary (Fig. 2) outside the inclusion. The algebraic system of equations, obtained after discretization of the boundary integral equations Eqns. (12)-(13) is of size 501.

The porelasticity of the half-space is described again by the Bardet model, having the same key material characteristics as those given above. The material properties of the elastic inclusion are considered as an elastic foundation with the following material characteristics: $\rho=2400.0 \mathrm{~kg} / \mathrm{m}^{3}, \lambda=0.12100000 \mathrm{E}+11 \mathrm{~N} / \mathrm{m}^{2}$, $\mu=0.18150000 \mathrm{E}+11 \mathrm{~N} / \mathrm{m}^{2}, \nu=0.2, C_{P}=4490.73 \mathrm{~m} / \mathrm{s}, C_{S V}=2750.0 \mathrm{~m} / \mathrm{s}$. An incident time-harmonic plane $\mathrm{P}$-wave with frequency $\omega$ is propagating in the plane $O x z$ with incident angle $\theta=\pi / 2$, according to axis $O x$.

Frequency dependent synthetic seismograms $\left|u_{x}\right|,\left|u_{y}\right|,\left|u_{z}\right|$ for observer point $B_{1}$ with coordinates $(2.5,-2.5,0)$ along the free surface of the elastic foundation, rested on dry or saturated half-space, subjected to incident $\mathrm{P}$-wave with incident angle $\theta=\pi / 4$ with respect to the axis $O x$ are presented in Figs, 8 a, b, c. The Poisson's ratio is $\nu_{\text {dry }}=0.4$ and the porosity takes the value of $n=0.1$ or 0.34 , while the foundation's depth is equal to $3 \mathrm{~m}$ or $2.5 \mathrm{~m}$.

From Figs. $8 \mathrm{a}, \mathrm{b}$ and $\mathrm{c}$ it can be seen, that the seismic response is very sensitive to the foundation depth and this effect depends on the frequency of the seismic excitation. For high values of the foundation depth $(d=3 \mathrm{~m})$, the displacement distribution curve with frequency variation is jagged compared with the lower value of $d=2.5 \mathrm{~m}$, that leads to a smooth displacement distribution. Moreover and much more important for practical design, the effect of the influence of the foundation depth on the seismic signal, recorded at free surface becomes even stronger for higher values of porosity in both cases, for dry and saturated soils.

It is shown, that the seismic signals depend strongly on the poroelastic behaviour of the soil. High values of porosity can significantly modify not only quantitatively but also qualitatively the seismic response. Pronounced oscillating behaviour of the seismic signal is observed for high frequency values. Furthermore, significant differences can be noticed at the seismic signals when they are recorded for a saturated and dry geological profile.

The obtained results show that not in all cases the saturated case leads to lower displacement amplitude. The seismic response in saturated domains depends also on additional factors, such as the frequency of the seismic excitation, the geometry of the heterogeneity and other key factors of the seismic scenario. 


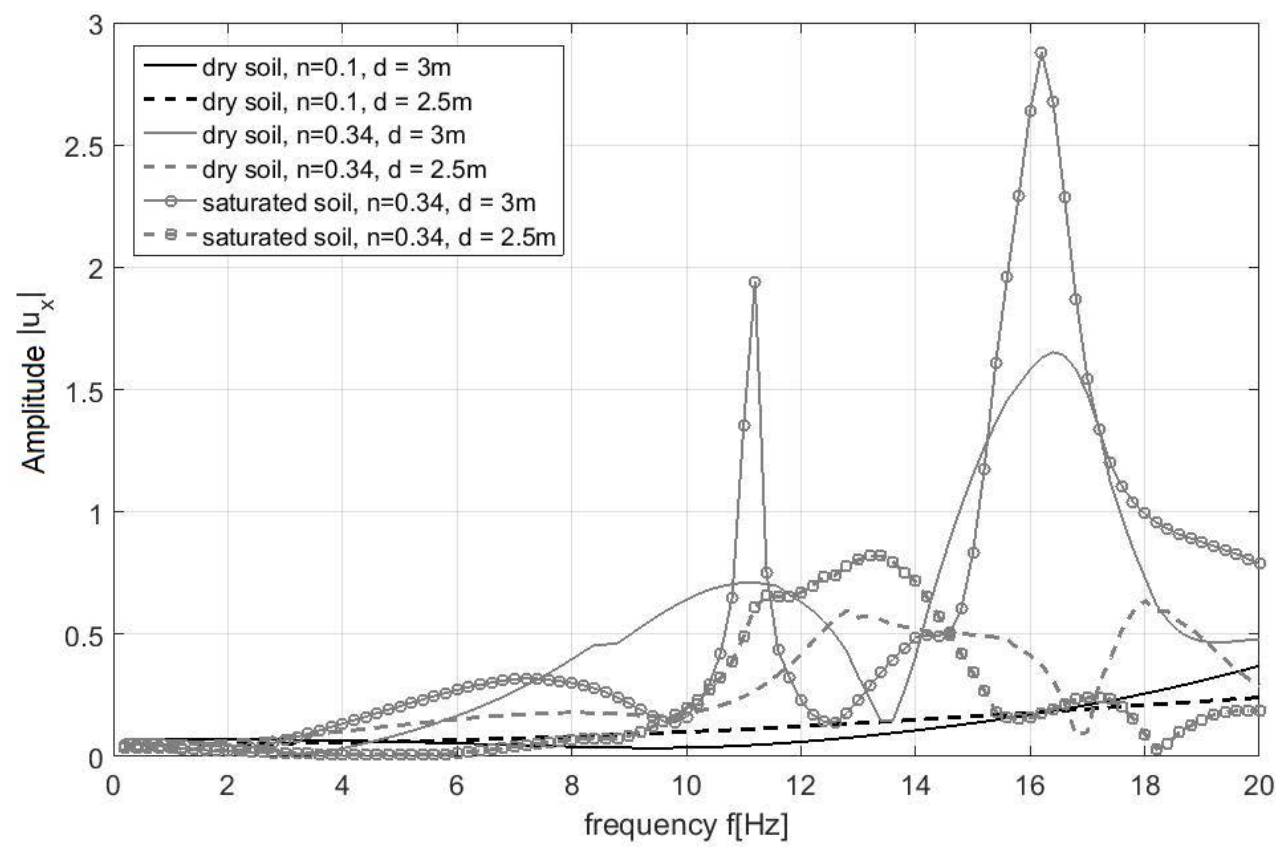

(a)

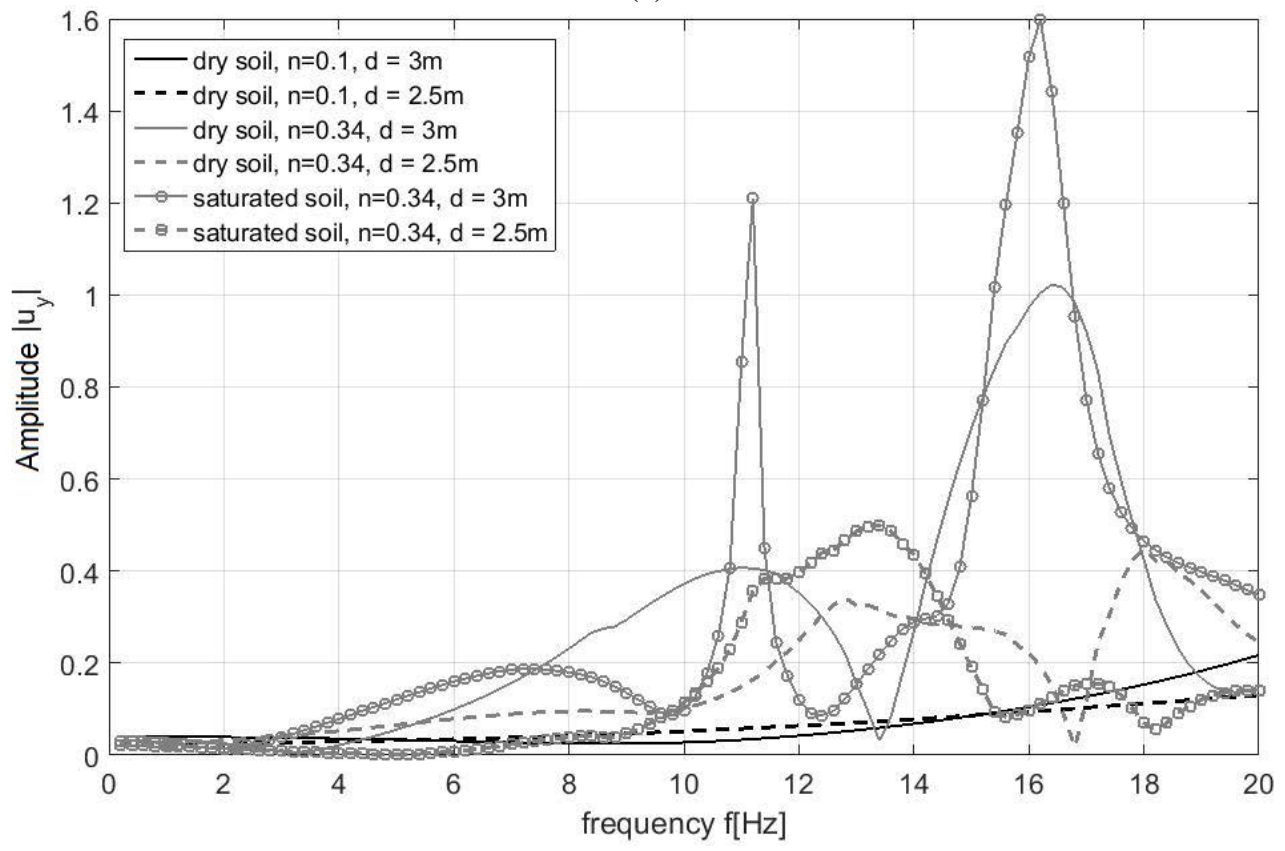

(b) 


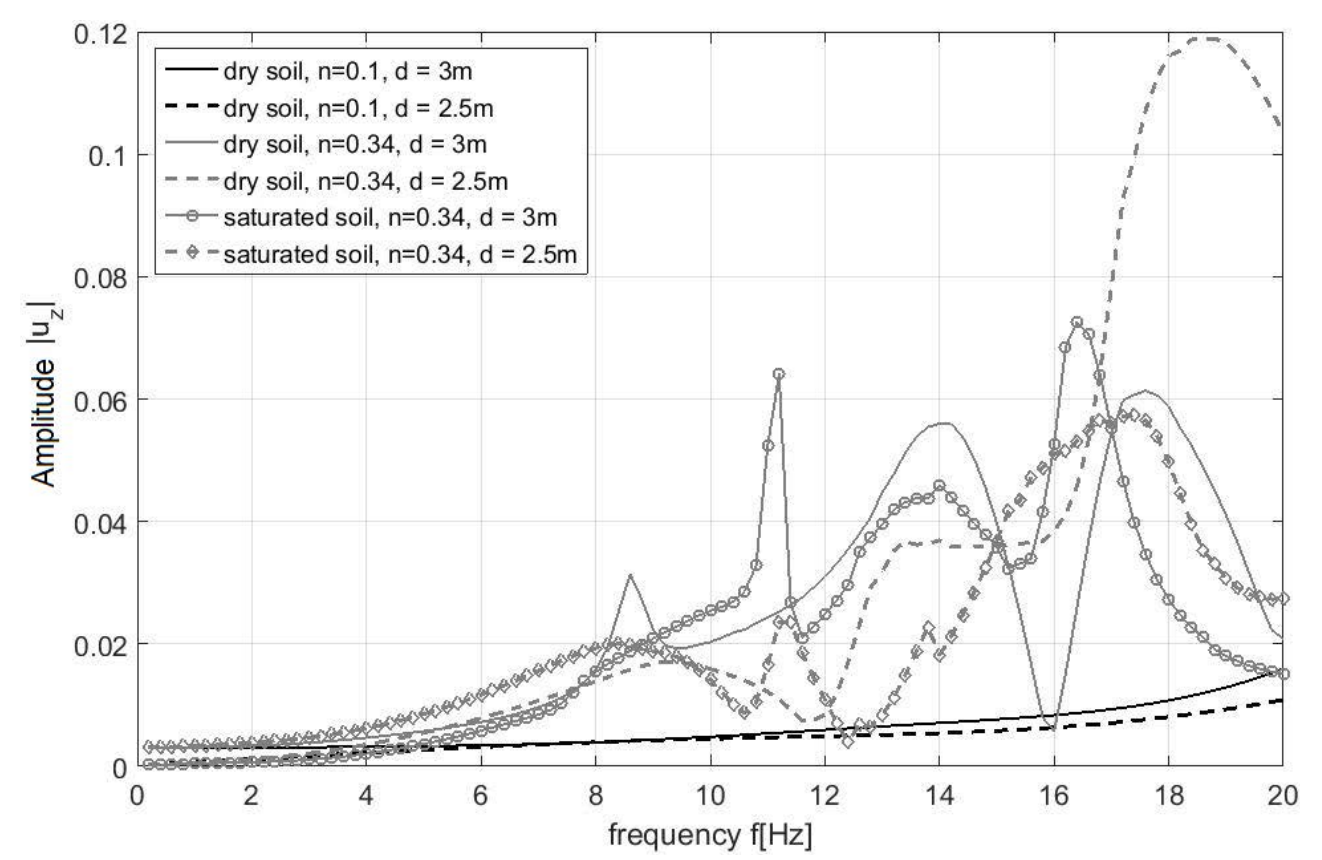

(c)

Fig. 8. Displacement amplitudes at point $B_{1}(2.5,-2.5,0)$ along the elastic foundation of depth $d=3 \mathrm{~m}$ or $d=2.5 \mathrm{~m}$, rested in dry and saturated half-space with Poisson's ratio $\nu_{\text {dry/saturated }}=0.4$ and porosity $n=0.1$ or $n=0.34$ versus frequency of incident P-wave with incident angle $\theta=\pi / 4$ : (a) amplitude $\left|u_{x}\right|$; (b) amplitude $\left|u_{y}\right|$; (c) amplitude $\left|u_{z}\right|$.

\section{CONCLUSions}

This work investigates 3D seismic wave propagation problems in poroelastic geomaterials to analyze the resulting seismic site effects in a homogeneous fluid saturated half-space with elastic heterogeneities and surface topography, based on the viscoelastic approximation of the Biot's model. It is also shown, that the Bardet onephase viscoelastic model is able to approximate and simulate the dynamic behaviour of poroelastic media well and deliver an excellent base for more realistic site studies.

The proposed numerical technique benefits from combining efficiently the advantages of the viscoelastic isomorphism with those of the BIEM itself, when it comes to handling of 3D wave propagation and scattering problems in semi-infinite heterogeneous poroelastic domains. The simulation results reveal that the seismic field in a poroelastic half-space with a $3 \mathrm{D}$ heterogeneity is sensitive to the stiffness and the Poisson's ratio of the solid skeleton of saturated soil. The elastic bulk module $K_{\text {dry }}$ is much greater than the fluid bulk module $K_{\mathrm{f}}\left(K_{\mathrm{dry}} \gg K_{\mathrm{f}}\right)$ at low porosities. Such 
a material is close to the elastic solid dominated case, where the dry frame strength is high and the fluid has no effect on the solid-fluid system behaviour. The greatest influence of porosity (associated with the fluid phase) can be observed for high porosity values where $K_{\mathrm{dry}} \approx K_{\mathrm{f}}$. Geomaterial for which is satisfied the condition $K_{\text {dry }} \ll K_{\mathrm{f}}$ is a fluid dominated material, where the portion of pore space is too large to form sustainable dry frame. More sensitive to the Poisson's ratio variations are the poroelastic materials, for which is satisfied the condition $K_{\text {dry }} \geq K_{\mathrm{f}}$. Poisson's ratio represents the consolidation status of the solid-skeleton, having in mind that its lower values concerns consolidated solid skeleton, while higher values unconsolidated one.

The numerical results show that the presence of water in the poroelastic material results in damping of the seismic waves. This phenomenon has been experimentally proved by Badiey et al. [52] and Oqushwitz [53]. The physical reasons for this effect may be (a) dissipation due to the viscoelastic behaviour of the skeleton or dissipation because of the fluid flow; (b) hardening effect, since the presence of fluid in the soil acts as material 'stiffener'. This effect can be explained by the used Bardet model. The pore pressure induced by the passage of seismic waves resists the compression and stiffens the rock. The shear strength of Biot's porous material is provided by the solid skeleton (i.e. the matrix) and it is unaffected by the fluid saturation, which implies $\mu_{\mathrm{sat}}=\mu_{\mathrm{dry}}$. The Lame coefficient $\lambda$, however, changes to $\lambda_{\mathrm{sat}}=\lambda_{\text {dry }}+$ $Q^{2} / R$, indicating a strengthening effect.

At this point, we stress that insofar as the Bardet model for one-phase materials is concerned, it cannot take into account the pore pressure boundary conditions along existing boundaries. Thus, there are limitations when the aforementioned model is applied to seismic analysis of poroelastic media, but it is still useful for engineering practice. More specifically, the viscoelastic-poroelastic similarity is useful for extracting approximate solutions, that can be used as benchmark cases to calibrate the accuracy of more advanced (in terms of constitutive laws) computational methods, applied to wave propagation problems in fluid-saturated soils.

The simulation results reveal, that the seismic wave field in a poroelastic heterogeneous half-space is a complex result of the mutual play of different key factors, such as the type and the characteristics of the seismic load, the wave-heterogeneity interaction, the type of heterogeneity and the poroelastic properties of the soil. The proposed approach has the potential to be extended and applied for dynamic soilstructure interaction problems, involving poroelastic media.

\section{ACKNOWLEDGEMENT}

The second author acknowledges the support of the Bilateral Bulgarian Greek (BG) Project-based Personnel Program between BAS and AUTH and BAS project DCD4/03.05.2017. 


\section{REFERENCES}

[1] Cen, Eurocode 8. Design Provisions of Structures for Earthquake Resistance. Part 1: General Rules, Seismic Actions and Rules for Buildings, Final Draft pr EN1998-1, European Committee for Standardization, Brussels, 2004.

[2] Moczo, P. Finite-difference Technique for SH Waves in 2-D Media using Irregular Grids: Application to the Seismic Response Problem. Geophys. J. Int., 99 (1989), 321329.

[3] Benites, R., K. AKI, K. Yomigida. Multiple Scattering of SH Waves in 2 D Media with Many Cavities. Pure and Applied Geophysics, 138 (1992), 353-390.

[4] YU, M. C., M. DRAVINSKI. Scattering of Plane Harmonic P, SV or Rayleigh Waves by a Completely Embedded Corrugated Cavity. Geophysical Journal International, 178 (2009), No. 1, 479-487.

[5] Dravinski, M., M. C. YU. Scattering of Plane Harmonic SH-waves by Multiple Inclusions. Geophysical Journal International, 186 (2011), No. 3, 1331-1346.

[6] Dravinski, M., M. C. YU. The Effect of Impedance Contrast upon Surface Motion due to Scattering of Plane Harmonic P, SV, and Rayleigh Waves by a Randomly Corrugated Elastic Inclusion. Journal of Seismology, 17 (2013), 281-295.

[7] Parvanova, S., P. Dineva, I.-K. Fontara, F. Wuttke. Seismic Response of Laterally Inhomogeneous Geological Region by Boundary Integral Equations. Geophysical Journal International, 202 (2015), No. 1, 424-438.

[8] Schanz, M. Wave Propagation in Viscoelastic and Poroelastic Continua: A Boundary Element Approach, (Lecture Notes in Applied and Computational Mechanics), New York, Springer-Verlag, LLC, 2001.

[9] Sanchez-Sesma, F. J., R. MAdariaga, K. Irikura. An Approximate Elastic 2- D Green's Function for a Constant-gradient Medium. Geophysical Journal International, 146 (2001), 237-248.

[10] Fontara, I.-K., P. Dineva, G. Manolis, F. Wuttke. Numerical Simulation of Seismic Wave Field in Graded Geological Media Containing Multiple Cavities. Geophysical Journal International, 206 (2016), No. 2, 921-940.

[11] Manolis, G., P. Dineva, Ts. Rangelov, Fr. WuttKe. Seismic Wave Propagation in Non-Homogeneous Elastic Media by Boundary Elements, Series: Solid Mechanics and Its Applications, 240 (2016), 294 pages, Springer, ISBN 978-3-319-45205-0.

[12] Schanz, M. Poroelastodynamics: Linear Models, Analytical Solutions, and Numerical Methods. Appl Mech Rev, 62 (2009), No. 3, 030803, doi:10.1115/1.3090831.

[13] Gatmiri, B., M. Kamalian. On the Fundamental Solution of Dynamic Poroelastic Boundary Integral Equations in Time Domain. International Journal of Geomechanics, 2 (2002), No. 4, 381-398.

[14] Gatmiri, B., K.V. Nguyen. Time 2D Fundamental Solution for Saturated Porous Media with Incompressible Fluid. Communications in Numerical Methods in Engineering, 21 (2005), No. 3, 119-132. 
[15] Seyerafian, S., B. Gatmiri, A. Nourzad. Green Functions for a Continuously Nonhomogenous Saturated Media. Int. J. Computer Methods in Engineering and Sciences (CMES), 15 (2006), No. 2, 115-125.

[16] Gatmiri, B., H. Eslami. Scattering of Harmonic Waves by a Circular Cavity in a Porous Medium: Complex Functions Theory Approach. International Journal of Geomechanics, 7 (2007), No. 5, 371-381.

[17] Theodorakopoulos, D. D., D. E. Beskosa. Application of Biot's Poroelasticity to Some Soil Dynamics Problems in Civil Engineering. Soil Dyn Earthq Eng., 26 (2006), 666-679.

[18] Dominguez, J. Boundary Elements in Dynamics, Southampton, Computational Mechanics Publications, 1993.

[19] Albers, B., S. Savidis, H. E. Tasan, O. Von Estroff, M. Gehlken. BEM and FEM Results of Displacements in a Poroelastic Column. Int. J. Appl. Math. Comput. Sci., 22 (2012), No. 4, 883-96.

[20] Yamamoto, K., M. Kitahara. A Numerical Method for Wave Scattering in Poroelastic Media, In: Proceedings of JSCE, 21 (2004), 143S-158S.

[21] Lin, C. H., V. W. LeE, M. D. TrifunaC. The Reflection of Plane Waves in a Poroelastic Half-space Saturated with Inviscid Fluid. Soil Dynamics and Earthquake Engineering, 25 (2005), 205-223.

[22] Hasheminejad, S. M., R. Avazmohammadi. Harmonic Wave Diffraction by Two Circular Cavities in a Poroelastic Formation. Soil Dyn Earthq Eng., 27 (2007), No. 1, 29-41.

[23] Nenning, M., M. Schanz. Infinite Elements in a Poroelastodynamic FEM. Int J Numer Anal Methods Geomech, 35 (2011), No. 16, 1774-1800.

[24] Gonsalves, I. R., D. J. Shippy, F. J. Rizzo. Direct Boundary Integral Equations for Elastodynamics in 3-D Half-spaces. Comput. Mech., 6 (1990), No. 4, 279-292.

[25] Sanchez-Sesma, F. J., F. Luzon. Seismic Response of Three-dimensional Alluvial Valleys for Incident P, S, and Rayleigh Waves. Bull. Seismol. Soc. Am., 85 (1995), No. 1, 269-284.

[26] Tadeu, A., P. A. Mendes, J. Antonio. The Simulation of 3-D Elastic Scattering produced by Thin Rigid Inclusions using the Traction Boundary Element Method. Comput. Struct., 84 (2006), 2244-53.

[27] Galis, M., P. Moczo, J. KRistek. A 3-D Hybrid Finite-difference-finite-element Viscoelastic Modelling of Seismic Wave Motion. Geophys. J. Int., 175 (2008), 153184.

[28] Chaillat, S., M. Bonnet, J. F. Semblat. A New Fast Multi-domain BEM to Model Seismic Wave Propagation and Amplification in 3-D Geological Structures. Geophys. J. Int., 177 (2009), 509-531.

[29] Fu, Z. J., W. Chen, J. T. Chen, W. Z. Qu. Singular Boundary Method: Three Regularization Approaches and Exterior Wave Applications. CMES Comput. Model Eng., 99 (2014), 417-443. 
[30] Grasso, E., S. Chaillat, M. Bonnet, J. F. Semblat. Application of the Multilevel Time-harmonic Fast Multipole BEM to 3-D Visco-elastodynamics. Eng. Anal. Bound. Elem., 36 (2012), No. 5, 744-58.

[31] Chaillat, S., M. Bonnet. Recent Advances on the Fast Multipole Accelerated Boundary Element Method for 3D Time-harmonic Elastodynamics. Wave Motion, 50 (2013), 1090-1104.

[32] LIU, Z., F. WU, D. WANG. The Multi-domain FMM-IBEM to Model Elastic Wave Scattering by Three-dimensional Inclusions in Infinite Domain. Eng. Anal. Bound. Elem., 11 (2015), 95-105.

[33] NiU, Y., M. Dravinski. Direct 3-D BEM for Scattering of Elastic Waves in a Homogeneous Anisotropic Half-space. Wave Motion, 38 (2003), No. 2, 165-75.

[34] Zimmerman, C., M. Stern. Boundary Element Solutions of 3-D Wave Scatter Problems in a Poroelastic Medium. Eng. Anal. Bound. Elem., 12 (1993), No. 4, 223-40.

[35] Zhao, C. G., Z. HAN. Three-dimensional Scattering and Diffraction of Plane Rayleigh-waves by a Hemispherical Alluvial Valley with Saturated Soil Deposit. Chin. J. Geophys., 50 (2007), No. 3, 905-14 [in Chinese].

[36] Liu, X., S. Greenhalgh, B. Zhou. Scattering of Plane Transverse Waves by Spherical Inclusions in a Poroelastic Medium. Geophys. J. Int., 176 (2009), No. 3, 938-50.

[37] Cheng, A. H. D., E. Detournay. On Singular Integral Equations and Fundamental Solutions of Poroelasticity. Int. J. Solids Struct., 35 (1998), No. 34, 4521-4555.

[38] Ding, B., J. Jiang. BEM Calculation for Porodynamics. Appl. Math. Mech., 36 (2015), No. 1, 31-47.

[39] Ding, B., J. JiAng, J. Hu. The Treatment of BEM for Porodynamic Problems Subjected to a Force Source in Time-domain. Engineering Analysis with Boundary Elements, 67 (2016), 138-151.

[40] BA, Z., J. LiAng, X. MEI. 3-D Scattering of Obliquely Incident Plane SV Waves by an Alluvial Valley embedded in a Fl. Earthq. Sci., 26 (2013), No. 2, 107-16.

[41] LiU, ZH., L. LiU, J. LiAng, Y. ZHOU. An Indirect BEM to Model the 3D Scattering of Elastic Waves in a Fluid - Saturated Poroelastic Half-space. Eng. Anal. Bound. Elem., 66 (2016), 91-108.

[42] Morochnik, V., J. P. BARdet. Viscoelastic Approximation of Poroelastic Media for Wave Scattering Problems. Soil Dynamics and Earthquake Engineering, 15 (2016), 337-346.

[43] BARdet, J. P. A Viscoelastic Model for the Dynamic Behaviour of Saturated Poroelastic Soils. Transactions of the ASME, 59 (1992), 128-135.

[44] Biot, M. A. Theory of Propagation of Elastic Waves in a Fluid-saturated Porous Solid. I: Low-frequency Range. J. Acoust. Soc. Am., 28 (1956), No. 2, 168-178.

[45] BIOT, M. A. Theory of Propagation of Elastic Waves in a Fluid-saturated Porous Solid. II: High-frequency Range. J. Acoust. Soc. Am., 28 (1962), No. 2, 179-191.

[46] Mavko, G., T. Mukerji, J. Dvorkon. The Rock Physics Handbook, New York, Cambridge University Press, 1998. 
[47] Dineva, P., M. Datcheva, T. Schanz. BIEM for Seismic Wave Propagation in Fluid Saturated Multilayered Media, In: Schweiger HF (ed) Numerical Methods in Geotechnical Engineering, Proceedings of $6^{\text {th }}$ European Conference on Numerical Methods in Geotechnical Engineering, 6-8 Sept 2006. Taylor \& Francis, Graz, 257265.

[48] Gal, D., J. Dvorkin, A. Nur. A Physical Model for Porosity Reduction in Sandstones. Geophysics, 63 (1998), 454-459.

[49] Achenback, J. D. Wave Propagation in Elastic Solids, Elsevier Science Publ. B. V., 1973.

[50] Omidvar, B., M. Rahimian, S. A. Mohammadnejad. Three-dimensional Scattering of Plane Harmonic SH, SV, and P Waves in Multilayered Alluvial Valleys, Asian Journal of Civil Engineering. 1 (2010), No. 5, 605-626.

[51] Reinoso, E., L. C. WROBEL, H. PowEr. Three-dimensional Scattering of Seismic Waves from Topographical Structures. Soil Dynamics and Earthquake Engineering, 16 (1997), 41-61.

[52] BAdiey, M., A. Cheng, Y. Mu. From Geology to Geoacoustics: Evaluation of BiotStoll Sound Speed and Attenuation for Shallow Water Acoustics. Journal of the Acoustical Society of America, 103 (1998), 309-320.

[53] OQushwitz, P. R. Applicability of the Biot Theory. Journal of the Acoustical Society of America, 77 (1985), No. 2, 429-464.

\section{APPENDIX: FREE FIELD MOTION}

Consider the wave propagating in the plane $x^{\prime}-z^{\prime}$ with incident angle $\theta$ in respect to the axis $O x^{\prime}$. The position of the plane $x^{\prime}-z^{\prime}$ in respect to the reference coordinate system is defined by the angle $\theta_{h}$ between the reference plane $x-y$ and plane

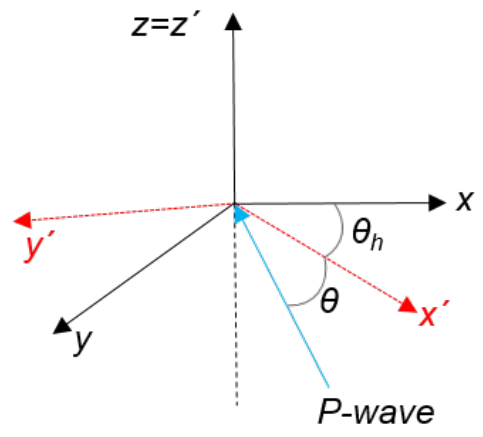

Fig. 9. Position of the wave propagation plane $x^{\prime}-z^{\prime}$ where the P-wave is propagating under incident angle $\theta$ in respect to the axis $O x^{\prime}, \theta_{h}$ is the angle between the reference plane $x-y$ and plane $x^{\prime}-y^{\prime}$. 
$x^{\prime}-y^{\prime}$. The displacement components in the reference coordinate system $O x y z$ are denoted by $\left(\begin{array}{lll}u_{x} & u_{y} & u_{z}\end{array}\right)^{T}$, while in the system $O x^{\prime} y^{\prime} z^{\prime}$ the displacement components are denoted by $\left(u_{x}^{\prime} u_{y}^{\prime} u_{z}^{\prime}\right)^{T}$. The relation between displacement components in both coordinate systems is as follows:

$$
\left(\begin{array}{l}
u_{x} \\
u_{y} \\
u_{z}
\end{array}\right)=T\left(\begin{array}{l}
u_{x}^{\prime} \\
u_{y}^{\prime} \\
u_{z}^{\prime}
\end{array}\right)=\left(\begin{array}{ccc}
\cos \theta_{h} & -\sin \theta_{h} & 0 \\
0 & 0 & 1 \\
\sin \theta_{h} & \cos \theta_{h} & 0
\end{array}\right)\left(\begin{array}{l}
u_{x}^{\prime} \\
u_{y}^{\prime} \\
u_{z}^{\prime}
\end{array}\right)
$$

$$
x^{\prime}=x \cos \theta_{h}+z \sin \theta_{h} ; \quad z^{\prime}=z .
$$

Note, that in the case $\theta_{h}=0, x^{\prime}=x, y^{\prime}=y, z^{\prime}=z$ and the wave propagates in the plane $x-z$, where

$$
\left(\begin{array}{l}
u_{x} \\
u_{y} \\
u_{z}
\end{array}\right)=\left(\begin{array}{c}
u_{x}^{\prime} \\
u_{y}^{\prime} \\
u_{z}^{\prime}
\end{array}\right) ; \quad T=\left(\begin{array}{ccc}
1 & 0 & 0 \\
0 & 0 & 1 \\
0 & 1 & 0
\end{array}\right) .
$$

Displacement of the free-field motion at any point of the half-space can be obtained by the following expression in the case $\theta_{h}=0$

$$
\begin{aligned}
u_{x}=A_{P}^{\text {in }}\left(\frac{k}{k_{P}}\right) e^{(-\nu z-i k x)} & +A_{P}^{\text {in }} R P P\left(\frac{k}{k_{P}}\right) e^{\left(\nu z-i k x_{1}\right)} \\
& +A_{P}^{\text {in }} R P S\left(\frac{i \nu^{\prime}}{k_{P}}\right) e^{\left(\nu^{\prime} z-i k x_{1}\right)}
\end{aligned}
$$

(A4) $\quad u_{y}=0$

$$
\begin{aligned}
u_{z}=A_{P}^{\text {in }}\left(-\frac{i \nu}{k_{P}}\right) e^{\left(-\nu z-i k x_{1}\right)} & +A_{P}^{\text {in }} R P P\left(\frac{i \nu}{k_{P}}\right) e^{\left(\nu z-i k x_{1}\right)} \\
& +A_{P}^{\text {in }} R P S\left(-\frac{k}{k_{P}}\right) e^{\left(\nu^{\prime} z-i k x_{1}\right)},
\end{aligned}
$$

where

$$
\begin{array}{lll}
k=k_{P} \cos \theta ; & \nu=i k_{P} \sin \theta ; \quad \nu^{\prime}=\sqrt{k^{2}-k_{S}^{2}} ; \\
k_{P}=\frac{\omega}{C_{P}} ; & k_{S}=\frac{\omega}{C_{S}} ; \quad & \Delta(k)=\left(2 k^{2}-k_{S}^{2}\right)^{2}-4 k^{2} \nu \nu^{\prime}
\end{array}
$$

$$
R P P=-\frac{\left(2 k^{2}-k_{S}^{2}\right)^{2}+4 k^{2} \nu \nu^{\prime}}{\Delta(k)} ; \quad R P S=-4 i k \nu \frac{\left(2 k^{2}-k_{S}^{2}\right)}{\Delta(k)}
$$


Traction of the free-field motion can be obtained by the following expression:

(A8) $t^{\mathrm{ff}}=$

$$
\left(\begin{array}{l}
{\left[\chi u_{x, x}^{\mathrm{ff}}+\lambda u_{y, y}^{\mathrm{ff}}+\lambda u_{z, z}^{\mathrm{ff}}\right] n_{x}+\mu\left(u_{x, y}^{\mathrm{ff}}+u_{y, x}^{\mathrm{ff}}\right) n_{y}+\mu\left(u_{x, z}^{\mathrm{ff}}+u_{z, x}^{\mathrm{ff}}\right) n_{z}} \\
\mu\left(u_{x, y}^{\mathrm{ff}}+u_{y, x}^{\mathrm{ff}}\right) n_{x}+\left[\chi u_{y, y}^{\mathrm{ff}}+\lambda u_{x, x}^{\mathrm{ff}}+\lambda u_{z, z}^{\mathrm{ff}}\right] n_{y}+\mu\left(u_{y, z}^{\mathrm{ff}}+u_{z, y}^{\mathrm{ff}}\right) n_{z} \\
\mu\left(u_{x, z}^{\mathrm{ff}}+u_{z, x}^{\mathrm{ff}}\right) n_{x}+\mu\left(u_{y, z}^{\mathrm{ff}}+u_{z, y}^{\mathrm{ff}}\right) n_{y}+\left[\chi u_{x, x}^{\mathrm{ff}}+\lambda u_{y, y}^{\mathrm{ff}}+\lambda u_{z, z}^{\mathrm{ff}}\right] n_{z}
\end{array}\right)
$$

with $\chi=(\lambda+2 \mu)$. 